\title{
Lifetime and current waterpipe use among adolescents in Tehran, Islamic Republic of Iran
}

A. Baheiraei, ${ }^{1,2}$ Z. Hamzehgardeshi, ${ }^{3,4}$ M.R. Mohammadi, ${ }^{5}$ S. Nedjat ${ }^{6}$ and E. Mohammadi ${ }^{7}$

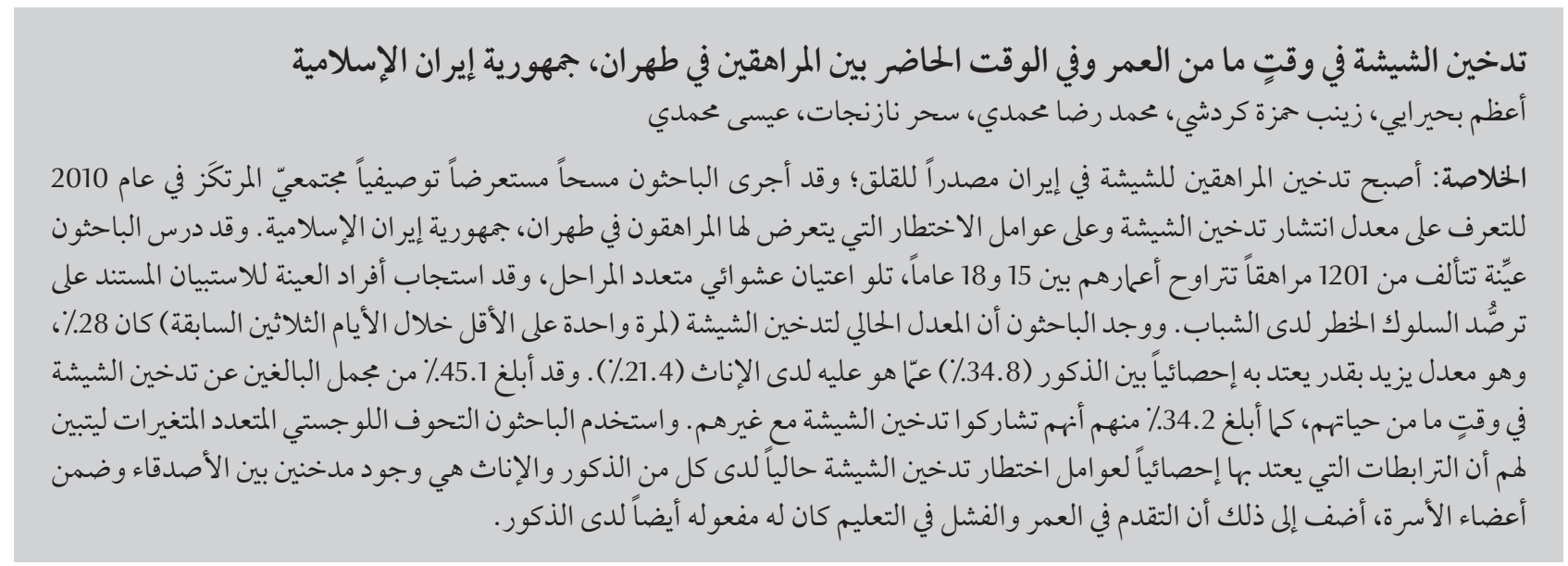

ABSTRACT Waterpipe use among Iranian adolescents has become a matter for concern. A descriptive, crosssectional community-based survey was performed in 2010 to determine the prevalence of waterpipe use and associated factors among Iranian adolescents in Tehran, Islamic Republic of Iran. After multi-stage, random cluster sampling 1201 adolescents aged 15-18 years old responded to a questionnaire based on the Youth Risk Behavior Surveillance. The prevalence of current waterpipe smoking (at least once in the previous 30 days) was $28.0 \%$, significantly higher among males (34.8\%) than females (21.4\%). A total of $45.1 \%$ of adolescents reported lifetime use (ever use) of waterpipes and $34.2 \%$ had ever shared a waterpipe. In multivariate logistic regression analysis the significant correlates of current waterpipe use for both males and females were having smokers among friends and family members, while for males, older age and educational failure were also risk factors.

Usage de la pipe à eau, présente ou passée, chez des adolescents de Téhéran (République islamique d'Iran)

RÉSUMÉ L'usage de la pipe à eau chez les adolescents iraniens est devenu préoccupant. Une enquête transversale descriptive a été menée dans la communauté en 2010 afin de déterminer la prévalence de l'usage de la pipe à eau et les facteurs associés chez des adolescents iraniens à Téhéran (République Islamique d'Iran). Dans un échantillonnage aléatoire en grappes à plusieurs degrés, 1201 adolescents âgés de 15 à 18 ans ont répondu à un questionnaire inspiré du Youth Risk Behavior Surveillance System (Système de surveillance des facteurs de risque chez les jeunes). La prévalence de l'usage de la pipe à eau au moment de l'étude (au moins une fois au cours des 30 derniers jours) était de 28,0 \%, mais la consommation des hommes était très supérieure à celle des femmes (34,8\% contre 21,4\%). Au total, 45,1\% des adolescents ont rapporté avoir déjà utilisé une pipe à eau dans leur vie et 34,2 \% déclaraient l'avoir déjà partagée avec d'autres. À l'analyse de régression logistique multivariée, les corrélats significatifs d'une utilisation actuelle de la pipe à eau pour les hommes comme pour les femmes étaient d'avoir des fumeurs parmi leurs amis et les membres de leur famille, tandis que pour les hommes, un âge plus avancé et l'échec scolaire étaient également des facteurs de risque.

${ }^{7}$ Department of Reproductive Health; ${ }^{2}$ Centre for Community-Based Participatory Research; ${ }^{5}$ Department of Psychiatric, Psychiatry and Psychology Research Centre; ${ }^{6}$ School of Public Health, Knowledge Utilization Research Centre, Tehran University of Medical Sciences, Tehran, Islamic Republic of Iran (Correspondence to Z. Hamzehgardeshi: Hamzeh@razi.tums.ac.ir).

${ }^{3}$ Traditional and Complementary Medicine Research Centre; ${ }^{4}$ Department of Midwifery and Reproductive Health, Mazandaran University of Medical Sciences, Sari, Islamic Republic of Iran.

'Department of Nursing, Tarbiat Modares University, Tehran, Islamic Republic of Iran.

Received: 13/09/12; accepted: 22/10/12 


\section{Introduction}

In recent years, the use of waterpipes for smoking tobacco has increased in many parts of the world, especially in the Eastern Mediterranean Region [1]. Waterpipe use is related to social, cultural and traditional factors and is more socially acceptable than cigarette use $[2,3]$. Previous studies have shown an $11 \%-32 \%$ prevalence of waterpipe smoking among youth in Middle Eastern countries, and recent observations have suggested that this proportion is increasing [4-10]. The results of the Global Youth Tobacco Survey (GYTS) showed that current waterpipe use among people aged $13-15$ years in the Middle East was 6\%-34\% [11]. Outside the Middle East, waterpipe use was reported to be $11 \%$ in Florida and $7 \%$ among 12th graders in Arizona in the United States of America (USA) $[12,13]$

Many waterpipe users believe that waterpipe smoking is less harmful than cigarette smoking $[14,15]$. Previous studies, however, have shown that it is addictive and is associated with notable health risks $[13,16-20]$. A recent systematic review of the effects of waterpipe smoking showed that it increases the risk of lung cancer, respiratory diseases and low birth weight by more than 2-fold [17]. Another systematic review reported that waterpipe use, similar to smoking cigarettes, contributes to the development of chronic obstructive pulmonary disease [20].

Waterpipe use among Iranian adolescents has become a matter for concern. Previous studies among Iranian adolescents showed that the prevalence of current waterpipe smoking was $25.7 \%$, with a higher prevalence among male than female respondents. Moreover, the prevalence of ever use and current use of waterpipes among adolescents had increased in 2005 compared with $2003[10,21]$. The present study was performed due to the lack of information available about the prevalence of waterpipe smoking and the associated factors among middle-age adolescents in developing countries, and the lack of populationbased studies examining waterpipe use among adolescents in the Islamic Republic of Iran. For example, a study was conducted in intermediate and high schools in Tehran municipal district 13 [21]. Another study reported waterpipe tobacco use among Iranian university students in 2 major universities in the south of the country [22].

Tehran with a population of 8 million people is the capital of Islamic Republic of Iran. Most of the Tehran population lives in the city and more than $50 \%$ of the Tehran population are aged $<25$ years. Since no previous studies have been based on adolescent populations in Tehran the trend of adolescent waterpipe use is unknown. Therefore, the current study was performed to determine the prevalence of waterpipe use and the associated factors in a study population representing 15-18-year-old adolescents in the metropolitan area of Tehran.

\section{Methods}

A population-based, cross-sectional survey was performed among 1201 adolescents in Tehran, Islamic Republic of Iran, in 2010.

\section{Sample and procedures}

The subjects were adolescents living in ordinary households in the 22 municipal districts of Tehran. The study population was derived through multistage cluster sampling. The blocks were considered as clusters and were randomly selected in proportion to the estimated population of each block; 10 households were selected from each cluster using systematic sampling. From each cluster, 10 households were selected in which 1 or more adolescent boys aged 15-18 years resided. A quota sampling method was used and for every female adolescent, a male adolescent was recruited. In addition, adolescents were assured that their information would remain confidential. The questionnaires were self-administered.

\section{Measures}

The research team developed a sociodemographic questionnaire and a waterpipe use questionnaire for use in this study. Waterpipe use was assessed with 3 items: "Have you ever smoked a waterpipe (even only 1 or 2 puffs)?”, "During the past 30 days, how many times have you used a waterpipe?" and "Have you ever shared a waterpipe?" The sociodemographic and waterpipe-use questionnaires were administered to adolescents.

Data used in the analysis included sociodemographic variables [23]; lifetime waterpipe use; current waterpipe use; waterpipe smoking on 20 or more of the 30 days before the survey; and waterpipe sharing. The overall prevalence of lifetime waterpipe use was defined as the percentage of adolescents who had any experience of ever using a waterpipe. Current waterpipe use was defined as the percentage of adolescents who had smoked on at least 1 day in the 30 days prior to the survey.

The lifetime and current waterpipe use were defined based on the Youth Risk Behaviors System Surveillance System [24]. Using principlefactor analysis, 12 economic variables (having a vacuum cleaner, separate kitchen, computer, washing machine, bath, freezer, dishwasher, private car, mobile phone, colour television, any type of video equipment and home telephone) were considered. The resulting variable was defined as wealth index and was divided into quintiles according to the percentage of items owned: $0 \%-20 \%$ (poorest), $21 \%-40 \%$ (poor), $41 \%-60 \%$ (intermediate), 61\%-80\% (rich) and 81\%-100\% (richest). 


\section{Statistical analysis}

The data gathered from the 1201 questionnaires were analysed using SPSS, version 16 for Windows and Stata, version 10. Descriptive indicators were determined. The prevalence rate was presented with $95 \%$ confidence intervals (CI). The chi-squared test was used for bivariate analysis. Univariate analysis was carried out, reporting crude odds ratios (OR) with 95\% CI. Variables with $P$-values of $<0.2$ were entered into a multivariate (backwards) logistic regression model to determine factors influencing waterpipe use. Lifetime and current waterpipe use were considered as dependent variables, and age, educational level, school type, social class, wealth index, parents' educational level, parental control on adolescent activity, use of punishment by parents, parents prefer sons to daughters, history of consulting about risk behaviours with experts, parental supervision on adolescent friend selection, family decision-making pattern, educational success, interest in education, adequacy of family income and having a waterpipe user among friends and family members were considered as independent variables. The results of the multivariate analysis were presented as adjusted $\mathrm{OR}$ with $95 \% \mathrm{CI}$ and $P$-values. In all analyses, $P \leq 0.05$ was taken to indicate statistical significance.

\section{Results}

\section{Prevalence of lifetime and current waterpipe use}

Of the 1201 adolescents included in this study, 535 (45.1\%) reported lifetime (ever use) of the waterpipe (Table 1). Prevalence of lifetime waterpipe use among male respondents was nearly double that of females $(\mathrm{OR}=1.66$; $P<$ 0.05 ). Among the $45.1 \%$ of adolescents who had experienced waterpipe smoking, $64.2 \%$ were current waterpipe users. The prevalence of having ever shared a waterpipe among the current waterpipe users was $79.5 \%$. Among these adolescents, sharing a waterpipe with friends was the most prevalent type of activity.

The prevalence of current waterpipe smoking among the total participants was $28.0 \%$ and was higher among male (34.8\%) than female (21.4\%) adolescents $(\mathrm{OR}=1.74 ; P<0.001)$. The prevalence of waterpipe smoking on 20 or more of the 30 days before the survey was $3.4 \%$ among all participants (5.4\% among males and $1.5 \%$ among females) $(\mathrm{OR}=2.96 ; P<0.01)$. The prevalence of waterpipe sharing was $34.2 \%$ among all participants (39.7\% among males and $28.9 \%$ among females $)(P=0.65)$.

\section{Bivariate analysis}

Tables 2 and 3 present the sociodemographic characteristics of the adolescents by sex and lifetime and current waterpipe use. The mean age of the adolescents was 16.7 (SD 1.1) years.

\section{Lifetime waterpipe use}

The results of the bivariate analysis in the female respondents indicated significant relationships between lifetime waterpipe use and educational failure $(P<0.05)$, being poor or poorest wealth index $(P<0.01)$, high levels of parental control $(P<0.01)$, very low or high levels of parental supervision $(P<0.05)$, the use of punishment by their parents $(P<0.01)$ and having smokers among family members $(P<0.01)$ and friends $(P<0.01)$ (Table 2). For the male adolescents there were significant relationships between lifetime waterpipe use and older age $(P<0.01)$, dropping out of school $(P<0.01)$, educational failure $(P<0.01)$, lack of interest in education $(P<0.01)$, adequacy of family income $(P<0.05)$, low or high levels of parental control $(P<0.05)$, decision-making in the family by people other than the parents and children $(P<0.05)$ and having smokers among family members $(P<$ $0.01)$ and friends $(P<0.01)$ (Table 2$)$.

\section{Current waterpipe use}

The results of the bivariate analysis in the female respondents showed significant relationships between current waterpipe

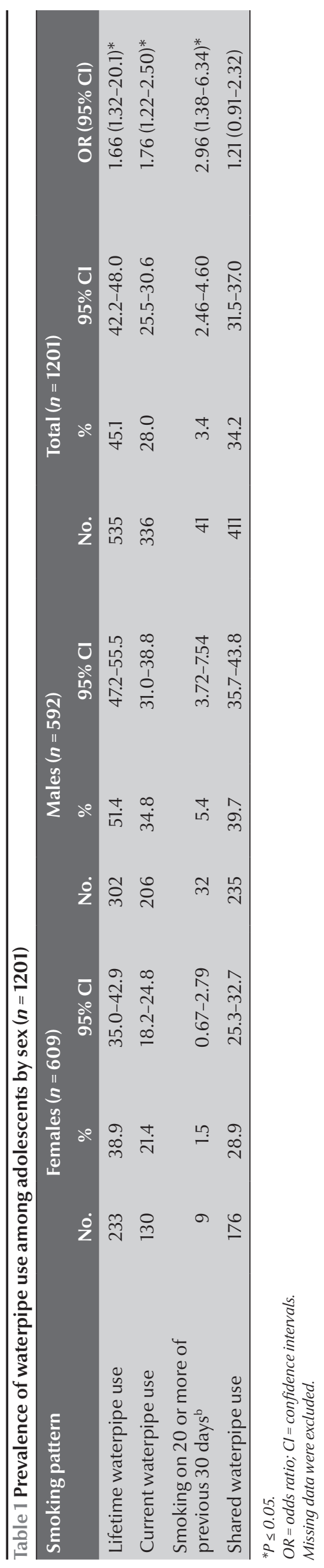




\begin{tabular}{|c|c|c|c|c|c|c|c|c|c|c|}
\hline \multirow[t]{3}{*}{ Variable } & \multicolumn{5}{|c|}{ Lifetime waterpipe use in females } & \multicolumn{5}{|c|}{ Lifetime waterpipe use in males } \\
\hline & \multicolumn{2}{|c|}{ No } & \multicolumn{2}{|c|}{ Yes } & \multirow[t]{2}{*}{ Crude OR $(95 \% \mathrm{CI})$} & \multicolumn{2}{|c|}{ No } & \multicolumn{2}{|c|}{ Yes } & \multirow[t]{2}{*}{ Crude OR $(95 \% \mathrm{Cl})$} \\
\hline & No. & $\%$ & No. & $\%$ & & No. & $\%$ & No. & $\%$ & \\
\hline \multicolumn{11}{|l|}{ Age (years) } \\
\hline 15 & 68 & 18.6 & 39 & 16.7 & 1 & 101 & 35.3 & 35 & 11.6 & 1 \\
\hline 16 & 84 & 23.0 & 44 & 18.9 & $0.91(0.53-1.65)$ & 75 & 26.2 & 54 & 17.9 & $2.08(1.24-3.49)^{* *}$ \\
\hline 17 & 100 & 27.3 & 72 & 30.9 & $1.26(0.76-2.06)$ & 60 & 21.0 & 74 & 24.5 & $3.56(2.13-5.95)^{* *}$ \\
\hline 18 & 114 & 31.1 & 78 & 33.5 & $1.19(0.73-1.94)$ & 50 & 17.5 & 139 & 46.0 & $8.02(4.86-13.3)^{* *}$ \\
\hline \multicolumn{11}{|l|}{ Educational status } \\
\hline Student & 331 & 91.9 & 214 & 94.7 & 1 & 267 & 94.3 & 263 & 88.0 & 1 \\
\hline Dropout & 29 & 8.1 & 12 & 5.3 & $0.71(0.35-1.45)$ & 16 & 5.7 & 36 & 12.0 & $2.44(1.30-4.56)^{* *}$ \\
\hline \multicolumn{11}{|l|}{ School type } \\
\hline Public & 237 & 72.9 & 145 & 69.0 & 1 & 135 & 51.7 & 155 & 59.4 & 1 \\
\hline Private & 88 & 27.1 & 65 & 31.0 & $1.08(0.68-1.72)$ & 126 & 48.3 & 106 & 40.6 & $0.71(0.47-1.06)$ \\
\hline \multicolumn{11}{|c|}{ Educational success } \\
\hline Yes & 253 & 75.3 & 184 & 82.9 & 1 & 244 & 84.2 & 188 & 67.4 & 1 \\
\hline No & 83 & 24.7 & 38 & 17.1 & $0.63(0.41-0.97)^{*}$ & 42 & 15.8 & 91 & 32.6 & $2.58(1.71-3.91)^{* *}$ \\
\hline \multicolumn{11}{|c|}{ Interest in education } \\
\hline Yes & 223 & 69.5 & 154 & 71.6 & 1 & 214 & 81.7 & 150 & 59.3 & 1 \\
\hline No & 98 & 30.5 & 61 & 28.4 & $1.21(0.98-2.45)$ & 48 & 18.3 & 113 & 40.7 & $2.73(1.80-4.14)^{* *}$ \\
\hline \multicolumn{11}{|l|}{ Social class } \\
\hline Upper & 2 & 0.6 & 2 & 0.9 & 1 & 0 & 0.0 & 4 & 1.4 & 1 \\
\hline Middle & 234 & 65.9 & 180 & 79.3 & $0.77(0.11-5.51)$ & 193 & 68.9 & 204 & 69.4 & $0.34(0.06-1.93)$ \\
\hline Working & 94 & 26.5 & 37 & 16.3 & $0.39(0.50-2.90)$ & 74 & 26.4 & 63 & 21.4 & $0.21(0.04-1.04)$ \\
\hline Lower & 25 & 7.0 & 8 & 3.5 & $0.27(0.03-2.36)$ & 13 & 4.6 & 23 & 7.8 & $0.26(0.06-1.26)$ \\
\hline \multicolumn{11}{|l|}{ Wealth index } \\
\hline Richest & 81 & 22.9 & 34 & 15.1 & 1 & 60 & 21.2 & 55 & 19.7 & 1 \\
\hline Rich & 83 & 23.4 & 44 & 19.6 & $1.26(1.83-2.17)$ & 53 & 18.7 & 36 & 12.9 & $0.74(0.42-1.30)$ \\
\hline Intermediate & 45 & 12.7 & 28 & 12.4 & $1.48(0.80-2.75)$ & 37 & 13.1 & 42 & 15.1 & $1.24(0.70-2.20)$ \\
\hline Poor & 70 & 19.8 & 55 & 24.4 & $1.87(1.10-3.19)^{*}$ & 56 & 19.8 & 66 & 23.7 & $1.29(0.77-2.14)$ \\
\hline Poorest & 75 & 21.2 & 64 & 28.4 & $2.03(1.21-3.42)^{* *}$ & 77 & 27.2 & 80 & 28.7 & $1.13(0.70-1.83)$ \\
\hline \multicolumn{11}{|c|}{ Adequacy offamily income } \\
\hline Inadequate & 40 & 11.9 & 20 & 9.0 & 1 & 24 & 9.4 & 47 & 16.8 & 1 \\
\hline $\begin{array}{l}\text { Approximately } \\
\text { adequate }\end{array}$ & 211 & 62.8 & 142 & 64.0 & $0.95(0.64-1.41)$ & 156 & 60.9 & 151 & 54.8 & $0.91(0.62-1.33)$ \\
\hline Adequate & 85 & 25.3 & 60 & 27.0 & $0.71(0.38-1.33)$ & 76 & 29.7 & 81 & 29.0 & $1.84(1.03-3.29)^{*}$ \\
\hline \multicolumn{11}{|c|}{ Father's educational level } \\
\hline High school & 131 & 36.6 & 75 & 32.9 & 1 & 118 & 42.0 & 140 & 47.3 & 1 \\
\hline University & 80 & 22.3 & 69 & 30.1 & $1.54(0.99-2.36)$ & 94 & 33.5 & 76 & 25.7 & $0.69(0.47-1.02)$ \\
\hline Secondary & 74 & 20.7 & 45 & 19.7 & $1.08(0.68-1.73)$ & 44 & 15.7 & 39 & 13.2 & $0.76(0.46-1.24)$ \\
\hline Primary & 57 & 15.9 & 35 & 15.3 & $1.09(0.66-1.82)$ & 17 & 6.0 & 27 & 9.1 & $1.35(0.70-2.61)$ \\
\hline Illiterate & 16 & 4.5 & 5 & 2.2 & $0.56(0.20-1.58)$ & 8 & 2.8 & 14 & 4.7 & $1.49(0.60-3.68)$ \\
\hline \multicolumn{11}{|c|}{ Mother's educational level } \\
\hline High school & 132 & 37.0 & 90 & 39.3 & 1 & 111 & 39.8 & 132 & 44.1 & 1 \\
\hline University & 65 & 18.2 & 53 & 23.1 & $1.20(0.76-1.89)$ & 68 & 24.4 & 63 & 21.1 & $0.62(0.14-2.84)$ \\
\hline Secondary & 72 & 20.2 & 46 & 20.1 & $0.94(0.60-1.49)$ & 55 & 19.7 & 48 & 16.1 & $0.77(0.50-1.18)$ \\
\hline Primary & 63 & 17.6 & 32 & 14.0 & $0.75(0.45-1.24)$ & 25 & 9.0 & 35 & 11.7 & $0.72(0.46-1.15)$ \\
\hline Illiterate & 25 & 7.0 & 8 & 3.5 & $0.47(0.20-1.09)$ & 20 & 7.2 & 21 & 7.0 & $1.16(0.65-2.06)$ \\
\hline
\end{tabular}




\begin{tabular}{|c|c|c|c|c|c|c|c|c|c|c|}
\hline \multirow[t]{3}{*}{ Variable } & \multicolumn{5}{|c|}{ Lifetime waterpipe use in females } & \multicolumn{5}{|c|}{ Lifetime waterpipe use in males } \\
\hline & \multicolumn{2}{|c|}{ No } & \multicolumn{2}{|c|}{ Yes } & \multirow[t]{2}{*}{ Crude OR $(95 \% \mathrm{CI})$} & \multicolumn{2}{|c|}{ No } & \multicolumn{2}{|c|}{ Yes } & \multirow[t]{2}{*}{ Crude OR $(95 \% \mathrm{Cl})$} \\
\hline & No. & $\%$ & No. & $\%$ & & No. & $\%$ & No. & $\%$ & \\
\hline \multicolumn{11}{|c|}{$\begin{array}{l}\text { Family decision-making } \\
\text { pattern }\end{array}$} \\
\hline $\begin{array}{l}\text { Father \& } \\
\text { mother }\end{array}$ & 170 & 47.5 & 107 & 48.4 & 1 & 144 & 51.6 & 127 & 43.3 & 1 \\
\hline Entire family & 72 & 20.1 & 45 & 20.4 & $0.70(0.28-1.75)$ & 50 & 17.9 & 55 & 18.8 & $2.41(1.01-5.77)^{*}$ \\
\hline Other people & 16 & 4.5 & 7 & 3.2 & $0.99(0.64-1.55)$ & 8 & 2.9 & 17 & 5.8 & $1.25(0.79-1.96)$ \\
\hline Mother & 21 & 5.9 & 24 & 10.9 & $1.82(0.96-3.42)$ & 12 & 4.3 & 11 & 3.8 & $1.04(0.44-2.44)$ \\
\hline Father & 79 & 22.1 & 38 & 17.2 & $0.76(0.48-1.21)$ & 65 & 23.3 & 83 & 28.3 & $1.45(0.97-2.17)$ \\
\hline \multicolumn{11}{|l|}{ Parental control } \\
\hline Intermediate & 107 & 29.9 & 108 & 47.8 & 1 & 151 & 53.4 & 106 & 35.9 & 1 \\
\hline High & 230 & 64.2 & 101 & 44.7 & $0.64(0.31-0.64)^{* *}$ & 69 & 24.4 & 25 & 8.5 & $0.52(0.31-0.87)^{*}$ \\
\hline Low & 21 & 5.9 & 17 & 7.5 & $0.99(0.34-2.92)$ & 63 & 22.3 & 164 & 55.6 & $2.85(1.79-4.54)^{* *}$ \\
\hline \multicolumn{11}{|l|}{$\begin{array}{l}\text { Parental } \\
\text { supervision }\end{array}$} \\
\hline Intermediate & 162 & 45.1 & 99 & 44.0 & 1 & 122 & 43.4 & 99 & 33.1 & 1 \\
\hline Very high & 37 & 10.3 & 11 & 4.9 & $0.49(0.24-0.99)^{*}$ & 30 & 10.7 & 26 & 8.7 & 1.07 (0.59-1.92) \\
\hline High & 73 & 20.3 & 38 & 16.9 & $0.85(0.54-1.36)$ & 80 & 28.5 & 62 & 20.7 & $0.96(0.63-1.46)$ \\
\hline Low & 59 & 16.4 & 43 & 19.1 & $1.19(0.75-1.90)$ & 32 & 11.4 & 71 & 23.7 & $2.73(1.67-4.48)^{* *}$ \\
\hline Very low & 28 & 7.8 & 34 & 15.1 & $1.99(1.14-3.48)^{*}$ & 17 & 6.0 & 41 & 13.7 & $2.97(1.59-5.55)^{* *}$ \\
\hline \multicolumn{11}{|l|}{$\begin{array}{l}\text { Parents' use of } \\
\text { punishment }^{a}\end{array}$} \\
\hline No & 275 & 77.2 & 146 & 64.3 & 1 & 165 & 58.9 & 183 & 61.8 & 1 \\
\hline Yes & 81 & 22.8 & 81 & 35.7 & $0.53(0.37-0.77)^{* *}$ & 115 & 41.1 & 113 & 38.2 & $1.13(0.81-1.58)$ \\
\hline \multicolumn{11}{|l|}{$\begin{array}{l}\text { Parents prefer } \\
\text { sons to daughters }\end{array}$} \\
\hline No & 28 & 80.1 & 174 & 77.3 & 1 & 181 & 66.1 & 176 & 62.4 & 1 \\
\hline Yes & 70 & 19.9 & 51 & 22.7 & $1.18(0.78-1.77)$ & 93 & 33.9 & 106 & 37.6 & $1.17(0.83-1.66)$ \\
\hline \multicolumn{11}{|c|}{ History of consultation ${ }^{b}$} \\
\hline No & 246 & 71.9 & 145 & 65.9 & 1 & 166 & 59.7 & 192 & 67.4 & 1 \\
\hline Yes & 96 & 28.1 & 75 & 34.1 & $0.75(0.52-1.09)$ & 112 & 40.3 & 93 & 32.6 & 1.39 (0.99-1.97) \\
\hline \multicolumn{11}{|c|}{$\begin{array}{l}\text { Having waterpipe user (s) } \\
\text { among family }\end{array}$} \\
\hline No & 255 & 70.8 & 95 & 41.1 & 1 & 242 & 87.1 & 181 & 60.5 & 1 \\
\hline Yes & 105 & 29.2 & 136 & 58.9 & $3.48(2.46-4.92)^{* *}$ & 36 & 12.9 & 118 & 39.5 & $4.38(2.88-6.67)^{* *}$ \\
\hline \multicolumn{11}{|c|}{$\begin{array}{l}\text { Having waterpipe user(s) } \\
\text { among friends }\end{array}$} \\
\hline No & 231 & 63.8 & 69 & 30.1 & 1 & 149 & 52.7 & 58 & 19.4 & 1 \\
\hline Yes & 131 & 36.2 & 160 & 69.9 & $4.09(2.87-5.38)^{* *}$ & 134 & 47.3 & 241 & 80.6 & $4.62(3.19-6.69)^{* *}$ \\
\hline
\end{tabular}

${ }^{*} P \leq 0.05 ;{ }^{* *} P \leq 0.01 ; * * * P \leq 0.001$

${ }^{a}$ The most common type of punishment used by the parents was verbal punishment; ${ }^{b}$ Having consultation with a teacher or another adult about risk behaviours. Missing data were excluded.

$O R=$ odds ratio; $C l=$ confidence interval.

use and older age $(P<0.05)$, going to pri- the males bivariate analysis showed sigvate schools $(P<0.05)$ and having smokers among friends $(P<0.01)$ and family members $(P<0.01)$ (Table 3). Among nificant relationships between current waterpipe use and older age $(P<0.01)$, dropping out of school $(P<0.01)$, low levels of parental supervision $(P<0.05)$, educational failure $(P<0.01)$ and having smokers among family members $(P<$ $0.01)$ and friends $(P<0.001)$ (Table 3$)$. 


\begin{tabular}{|c|c|c|c|c|c|c|c|c|c|c|}
\hline \multirow[t]{3}{*}{ Variable } & \multicolumn{5}{|c|}{ Current waterpipe use in females } & \multicolumn{5}{|c|}{ Current waterpipe use in males } \\
\hline & \multicolumn{2}{|c|}{ No } & \multicolumn{2}{|c|}{ Yes } & \multirow[t]{2}{*}{ Crude OR $(95 \% \mathrm{CI})$} & \multicolumn{2}{|c|}{ No } & \multicolumn{2}{|c|}{ Yes } & \multirow[t]{2}{*}{ Crude OR $(95 \% \mathrm{Cl})$} \\
\hline & No. & $\%$ & No. & $\%$ & & No. & $\%$ & No. & $\%$ & \\
\hline \multicolumn{11}{|l|}{ Age (years) } \\
\hline 15 & 20 & 20.4 & 19 & 14.6 & 1 (ref.) & 20 & 22.5 & 13 & 6.3 & 1 (ref.) \\
\hline 16 & 21 & 21.4 & 21 & 16.2 & $2.28(1.03-5.04)^{*}$ & 15 & 16.9 & 39 & 18.9 & $4.00(1.60-10.0)^{* *}$ \\
\hline 17 & 33 & 33.7 & 38 & 29.2 & $1.21(0.55-2.65)$ & 20 & 22.5 & 52 & 25.2 & $4.00(1.68-9.53)^{* *}$ \\
\hline 18 & 24 & 24.5 & 52 & 40.0 & $1.05(0.44-2.52)$ & 34 & 38.2 & 102 & 49.5 & $4.62(2.08-10.3)^{* *}$ \\
\hline \multicolumn{11}{|l|}{ Educational status } \\
\hline Student & 96 & 99.0 & 116 & 92.8 & 1 (ref.) & 85 & 96.2 & 171 & 83.7 & 1 (ref.) \\
\hline Dropout & 1 & 1.0 & 9 & 7.2 & $7.45(0.93-9.84)$ & 3 & 3.4 & 33 & 16.2 & $5.47(1.63-8.37)^{* *}$ \\
\hline \multicolumn{11}{|l|}{ School type } \\
\hline Public & 72 & 75.0 & 72 & 64.9 & 1 (ref.) & 49 & 58.3 & 103 & 60.2 & 1 (ref.) \\
\hline Private & 24 & 25.0 & 39 & 35.1 & $2.50(1.12-5.58)^{*}$ & 35 & 41.7 & 68 & 39.8 & $1.08(0.57-2.04)$ \\
\hline \multicolumn{11}{|c|}{ Educational success } \\
\hline Yes & 84 & 88.4 & 99 & 79.8 & 1 (ref.) & 69 & 82.1 & 115 & 60.2 & 1 (ref.) \\
\hline No & 11 & 11.6 & 25 & 20.2 & $1.93(0.90-4.15)$ & 15 & 17.9 & 76 & 39.8 & $3.04(1.62-5.70)^{* *}$ \\
\hline \multicolumn{11}{|c|}{ Interest in education } \\
\hline Yes & 67 & 69.8 & 85 & 72.6 & 1 (ref.) & 56 & 68.3 & 91 & 54.8 & 1 (ref.) \\
\hline No & 29 & 30.2 & 32 & 27.4 & $1.93(0.90-4.15)$ & 26 & 31.8 & 75 & 45.1 & $1.73(0.96-3.13)$ \\
\hline \multicolumn{11}{|l|}{ Social class } \\
\hline Upper & 0 & 0.0 & 2 & 1.6 & 1 (ref.) & 1 & 1.1 & 3 & 1.5 & 1 (ref.) \\
\hline Middle & 79 & 81.4 & 97 & 77.6 & $0(0)$ & 59 & 67.8 & 140 & 69.7 & $0.79(0.08-7.60)$ \\
\hline Working & 16 & 16.5 & 20 & 16.0 & $0(0)$ & 21 & 24.1 & 41 & 20.4 & $0.65(0.06-6.65)$ \\
\hline Lower & 2 & 2.1 & 6 & 4.8 & $0(0)$ & 6 & 6.8 & 17 & 8.5 & $0.67(0.05-8.16)$ \\
\hline \multicolumn{11}{|l|}{ Wealth index } \\
\hline Richest & 16 & 16.7 & 17 & 13.6 & 1 (ref.) & 19 & 22.9 & 34 & 17.8 & 1 (ref.) \\
\hline Rich & 21 & 21.9 & 23 & 18.4 & $1.45(0.62-3.41)$ & 10 & 12.0 & 25 & 13.1 & $1.40(0.56-3.52)$ \\
\hline Intermediate & 15 & 15.6 & 13 & 10.4 & $1.65(0.69-3.96)$ & 9 & 10.8 & 33 & 17.3 & $2.05(0.81-5.18)$ \\
\hline Poor & 20 & 20.8 & 35 & 28.0 & $0.82(0.30-2.24)$ & 22 & 26.5 & 44 & 23.0 & $1.12(0.52-2.39)$ \\
\hline Poorest & 24 & 25.0 & 37 & 29.6 & $1.03(0.42-2.54)$ & 23 & 27.7 & 55 & 28.8 & $1.34(0.64-2.81)$ \\
\hline \multicolumn{11}{|c|}{$\begin{array}{l}\text { Adequacy offamily } \\
\text { income }\end{array}$} \\
\hline Inadequate & 9 & 9.5 & 12 & 9.8 & 1 (ref.) & 15 & 17.6 & 32 & 17.1 & 1 (ref.) \\
\hline $\begin{array}{l}\text { Approximately } \\
\text { adequate }\end{array}$ & 64 & 67.4 & 73 & 59.3 & $0.86(0.34-2.16)$ & 43 & 50.6 & 101 & 54.0 & $1.10(0.54-2.24)$ \\
\hline Adequate & 22 & 23.2 & 38 & 30.9 & $1.29(0.47-3.56)$ & 27 & 31.8 & 54 & 28.9 & $0.94(0.44-2.02)$ \\
\hline \multicolumn{11}{|c|}{ Father's educational level } \\
\hline High school & 39 & 39.8 & 36 & 28.6 & 1 (ref.) & 38 & 44.8 & 99 & 48.5 & 1 (ref.) \\
\hline University & 29 & 29.6 & 37 & 29.4 & $1.75(0.00-2.02)$ & 24 & 28.2 & 50 & 24.5 & $0.77(0.42-1.42)$ \\
\hline Secondary & 16 & 16.3 & 28 & 22.2 & $1.55(0.68-3.53)$ & 10 & 11.8 & 28 & 13.7 & $1.04(0.46-2.35)$ \\
\hline Primary & 14 & 14.3 & 20 & 15.9 & $1.90(0.88-4.09)$ & 10 & 11.8 & 16 & 7.8 & $0.59(0.25-1.43)$ \\
\hline Illiterate & 0 & 0.0 & 5 & 4.0 & $1.38(0.71-2.70)$ & 3 & 3.5 & 11 & 5.4 & $1.36(0.36-5.16)$ \\
\hline
\end{tabular}

\section{Multivariate logistic regression}

The results of the multivariate logistic regression analysis are shown in Tables 4 and 5 .

\section{Lifetime waterpipe use}

The results of the multivariate logistic regression analysis in the female respondents indicated significant relationships between lifetime waterpipe use and the use of punishment by the parents $(P<$ $0.01)$, history of consultation with an expert $(P<0.05)$, very low or high levels 


\begin{tabular}{|c|c|c|c|c|c|c|c|c|c|c|}
\hline \multirow[t]{3}{*}{ Variable } & \multicolumn{5}{|c|}{ Current waterpipe use in females } & \multicolumn{5}{|c|}{ Current waterpipe use in males } \\
\hline & \multicolumn{2}{|c|}{ No } & \multicolumn{2}{|c|}{ Yes } & \multirow[t]{2}{*}{ Crude OR $(95 \% \mathrm{CI})$} & \multicolumn{2}{|c|}{ No } & \multicolumn{2}{|c|}{ Yes } & \multirow[t]{2}{*}{ Crude OR $(95 \% \mathrm{Cl}$} \\
\hline & No. & $\%$ & No. & $\%$ & & No. & $\%$ & No. & $\%$ & \\
\hline \multicolumn{11}{|l|}{$\begin{array}{l}\text { Mother's } \\
\text { educational level }\end{array}$} \\
\hline High school & 40 & 41.2 & 49 & 38.9 & 1 (ref.) & 38 & 43.1 & 90 & 44.1 & 1 (ref.) \\
\hline University & 28 & 28.9 & 23 & 18.3 & $1.35(0.00-1.91)$ & 20 & 22.7 & 40 & 19.6 & $0.84(0.44-1.63)$ \\
\hline Secondary & 20 & 20.6 & 25 & 19.8 & $1.94(0.80-4.72)$ & 10 & 11.4 & 38 & 18.6 & $1.60(0.72-3.54)$ \\
\hline Primary & 9 & 9.3 & 21 & 16.7 & $1.04(0.51-2.14)$ & 15 & 17.0 & 20 & 9.8 & $0.56(0.26-1.21)$ \\
\hline Illiterate & 0 & 0.0 & 8 & 6.3 & $0.69(0.34-1.37)$ & 5 & 5.7 & 16 & 7.8 & $1.34(0.46-3.94)$ \\
\hline \multicolumn{11}{|l|}{$\begin{array}{l}\text { Family decision- } \\
\text { making pattern }\end{array}$} \\
\hline $\begin{array}{l}\text { Father and } \\
\text { mother }\end{array}$ & 52 & 54.2 & 53 & 44.2 & 1 (ref.) & 34 & 39.5 & 92 & 45.8 & 1 (ref.) \\
\hline Entire family & 23 & 24.0 & 23 & 19.2 & $2.45(0.46-3.21)$ & 17 & 19.8 & 36 & 17.9 & $0.68(0.23-1.98)$ \\
\hline Other people & 2 & 2.1 & 5 & 4.2 & $0.98(0.49-1.96)$ & 6 & 7.0 & 11 & 5.5 & $0.78(0.39-1.57)$ \\
\hline Mother & 7 & 7.3 & 14 & 11.7 & $1.96(0.73-5.25)$ & 4 & 4.7 & 7 & 3.5 & $0.65(0.18-2.35)$ \\
\hline Father & 12 & 12.5 & 25 & 20.8 & $2.04(0.93-4.49)$ & 25 & 29.1 & 55 & 27.4 & $0.81(0.44-1.50)$ \\
\hline \multicolumn{11}{|l|}{ Parental control } \\
\hline Intermediate & 40 & 41.7 & 63 & 50.4 & 1 (ref.) & 35 & 40.2 & 71 & 35.0 & 1 (ref.) \\
\hline High & 50 & 52.1 & 52 & 41.6 & $1.06(0.36-3.14)$ & 11 & 12.6 & 11 & 5.4 & $0.49(0.20-1.25)$ \\
\hline Low & 6 & 6.2 & 10 & 8.0 & $0.66(0.38-1.15)$ & 41 & 47.1 & 120 & 59.4 & $1.28(0.67-2.47)$ \\
\hline \multicolumn{11}{|l|}{$\begin{array}{l}\text { Parental } \\
\text { supervision }\end{array}$} \\
\hline Intermediate & 45 & 46.4 & 51 & 41.5 & 1 (ref.) & 32 & 36.4 & 65 & 31.9 & 1 (ref.) \\
\hline Very high & 3 & 3.1 & 7 & 5.7 & $1.12(0.51-2.46)$ & 11 & 12.5 & 13 & 6.4 & $0.58(0.24-1.44)$ \\
\hline High & 18 & 18.6 & 20 & 16.3 & $1.43(0.68-3.01)$ & 22 & 25.0 & 39 & 19.1 & $0.87(0.45-1.71)$ \\
\hline Low & 16 & 16.5 & 26 & 21.1 & $0.98(0.46-2.08)$ & 12 & 13.6 & 59 & 28.9 & $2.42(1.14-5.13)^{*}$ \\
\hline Very low & 15 & 15.5 & 19 & 15.4 & $2.06(0.50-8.44)$ & 11 & 12.5 & 28 & 13.7 & $1.25(0.55-2.83)$ \\
\hline \multicolumn{11}{|l|}{$\begin{array}{l}\text { Parents' use of } \\
\text { punishment }^{a}\end{array}$} \\
\hline No & 65 & 66.3 & 80 & 64.0 & 1 (ref.) & 54 & 62.1 & 124 & 61.4 & 1 (ref.) \\
\hline Yes & 33 & 33.7 & 45 & 36.0 & $0.90(0.52-1.57)$ & 33 & 37.9 & 78 & 38.6 & $0.97(0.58-1.63)$ \\
\hline \multicolumn{11}{|l|}{$\begin{array}{l}\text { Parents prefer } \\
\text { sons to daughters }\end{array}$} \\
\hline No & 76 & 78.4 & 93 & 76.2 & 1 (ref.) & 54 & 65.9 & 118 & 60.8 & 1 (ref.) \\
\hline Yes & 21 & 21.6 & 29 & 23.8 & $1.13(0.60-2.14)$ & 28 & 34.1 & 76 & 39.2 & $1.24(0.72-2.13)$ \\
\hline \multicolumn{11}{|c|}{ History of consultation } \\
\hline No & 61 & 65.6 & 79 & 65.3 & 1 (ref.) & 56 & 68.3 & 132 & 67.0 & 1 (ref.) \\
\hline Yes & 32 & 34.4 & 42 & 34.7 & $0.99(0.56-1.74)$ & 26 & 31.7 & 65 & 33.0 & $0.94(0.54-1.64)$ \\
\hline \multicolumn{11}{|c|}{$\begin{array}{l}\text { Having waterpipeuser(s) } \\
\text { among family }\end{array}$} \\
\hline No & 56 & 58.3 & 40 & 30.8 & 1 (ref.) & 66 & 75.9 & 113 & 55.1 & 1 (ref.) \\
\hline Yes & 40 & 41.7 & 90 & 69.2 & $3.15(1.82-5.46)^{* *}$ & 21 & 24.1 & 92 & 44.9 & $2.56(1.46-4.49)^{* *}$ \\
\hline \multicolumn{11}{|c|}{$\begin{array}{l}\text { Having waterpipeuser (s) } \\
\text { among friends }\end{array}$} \\
\hline No & 37 & 38.5 & 32 & 25.0 & 1 (ref.) & 29 & 33.3 & 26 & 12.7 & 1 (ref.) \\
\hline Yes & 59 & 61.5 & 96 & 75.0 & $1.88(1.06-3.34)^{* *}$ & 58 & 66.7 & 179 & 87.3 & $3.44(1.88-6.31)^{* * *}$ \\
\hline
\end{tabular}

${ }^{*} P \leq 0.05 ;{ }^{* *} P \leq 0.01 ;{ }^{* *} P \leq 0.001$.

${ }^{a}$ The most common type of punishment used by the parents was verbal punishment.

Missing data were excluded.

$O R=$ odds ratio; $C l=$ confidence interval. 
of parental supervision $(P<0.05)$ and having smokers among family members $(P<0.01)$ and friends $(P<0.01)$ (Table 4). Among the male respondents there were significant relationships between lifetime waterpipe use and older age $(P$ $<0.01$ ), low levels of parental control $P<0.01)$, history of consultation with an expert $(P<0.05)$, very low or high levels of parental supervision $(P<0.05)$ and having smokers among friends $(P$ $<0.01)$ and family members $(P<0.01)$ (Table 4).

\section{Current waterpipe use}

The results of the multivariate logistic regression analysis in the female respondents indicated that having smokers among friends $(P<0.05)$ and family members $(P<0.01)$ was significantly related to current waterpipe use. In the male respondents, current waterpipe use was associated with older age $(P<$ $0.05)$, educational failure $(P<0.05)$, and having smokers among friends $(P$ $<0.01)$ and family members $(P<0.01)$ (Table 5).

\section{Discussion}

In this study, almost half the adolescent respondents had ever used a waterpipe for smoking tobacco. The overall prevalence of current waterpipe use was $28.0 \%$. Ever-use of waterpipes and current waterpipe smoking was significantly higher among male than female respondents.

In a previous study of Iranian adolescents, the prevalence of current waterpipe use was $25.7 \%$ [21]. The higher prevalence of waterpipe smoking in the present study may be a warning sign suggesting an increased use of tobacco productsother than cigarettes, especially waterpipes, by adolescents. The results of the present study were compatible with those of the Global Youth Tobacco Survey (GYTS), which indicated an increasing prevalence of tobacco use in developing countries [25]. Our study also indicated that the prevalence

\begin{tabular}{|c|c|c|}
\hline \multicolumn{3}{|c|}{$\begin{array}{l}\text { Table } 4 \text { Results of the multivariate logistic regression analysis of lifetime } \\
\text { waterpipe use in adolescents using the sociodemographic variables in a backward } \\
\text { regression model }\end{array}$} \\
\hline \multirow[t]{2}{*}{ Variable } & \multicolumn{2}{|c|}{ Adjusted OR (95\% CI) } \\
\hline & Females & Males \\
\hline \multicolumn{3}{|l|}{ Age (years) } \\
\hline 15 & $\mathrm{n} / \mathrm{s}$ & 1 (ref.) \\
\hline 16 & & $1.34(0.65-2.79)$ \\
\hline 17 & & $1.58(0.75-3.35)$ \\
\hline 18 & & $5.12(2.43-10.82)^{* *}$ \\
\hline \multicolumn{3}{|l|}{ Parental control } \\
\hline Intermediate & $\mathrm{n} / \mathrm{s}$ & 1 (ref.) \\
\hline High & & $0.62(0.30-1.90)$ \\
\hline Low & & $2.32(1.17-4.60)^{* *}$ \\
\hline \multicolumn{3}{|c|}{ Parental supervision } \\
\hline Intermediate & 1 (ref.) & 1 (ref.) \\
\hline Very high & $0.36(0.15-0.92)^{*}$ & $2.51(1.10-6.30)^{*}$ \\
\hline High & $1.07(0.61-1.89)$ & $1.45(0.77-2.72)$ \\
\hline Low & $1.21(0.68-2.16)$ & $3.47(1.63-7.42)^{* *}$ \\
\hline Very low & $2.42(1.21-4.43)^{*}$ & $1.05(0.42-2.65)$ \\
\hline \multicolumn{3}{|c|}{ Use of punishment } \\
\hline No & 1 (ref.) & $\mathrm{n} / \mathrm{s}$ \\
\hline Yes & $0.51(0.32-0.81)^{* *}$ & \\
\hline \multicolumn{3}{|c|}{ History of consultation } \\
\hline No & 1 (ref.) & 1 (ref.) \\
\hline Yes & $0.54(0.32-0.93)^{*}$ & $1.81(1.06-3.09)^{*}$ \\
\hline \multicolumn{3}{|c|}{$\begin{array}{l}\text { Having waterpipe user(s) among } \\
\text { family }\end{array}$} \\
\hline No & 1 (ref.) & 1 (ref.) \\
\hline Yes & $3.43(2.24-5.24)^{* *}$ & $2.72(1.57-4.70)^{* *}$ \\
\hline \multicolumn{3}{|c|}{$\begin{array}{l}\text { Having waterpipe user(s) among } \\
\text { friends }\end{array}$} \\
\hline No & 1 (ref.) & 1 (ref.) \\
\hline Yes & $2.82(1.86-4.29)^{* *}$ & $3.81(2.07-7.03)^{* *}$ \\
\hline
\end{tabular}

${ }^{*} P \leq 0.05 ;{ }^{*} P \leq 0.01$.

$\mathrm{n} / \mathrm{s}=$ not significant in model; $\mathrm{OR}=$ odds ratio; $\mathrm{Cl}=$ confidence interval .

of current waterpipe use among Iranian adolescents was higher than previously reported. Previous studies have shown an $11 \%-32 \%$ prevalence of waterpipe smoking among youth in Middle Eastern countries, and recent observations have suggested that this proportion is increasing $[4,5,7-9,12,26-28]$. In the present study, the male to female ratio of lifetime waterpipe use was approximately 1.7:1 $(\mathrm{OR}=1.66)$. Consistent with other studies, male sex is a predictor of tobacco use [29]. The GYTS also showed a tendency towards females' increasing use of tobacco products other than cigarettes, such as waterpipes $[11,30]$.

Furthermore, the results of the current study were consistent with those of a study performed among high-school students in the USA, showing that waterpipe smoking was more common among older adolescents [12]. This finding reflects the social and environmental changes in adolescence [24]. Consistent with other studies, increasing age was a predictor of tobacco use [31]. In this study, older age was the 


\begin{tabular}{|c|c|c|}
\hline \multicolumn{3}{|c|}{$\begin{array}{l}\text { Table } 5 \text { Results of the multivariate logistic regression analysis of current waterpipe } \\
\text { use in adolescents using the sociodemographic variables in a backward regression } \\
\text { model }\end{array}$} \\
\hline \multirow[t]{2}{*}{ Variable } & \multicolumn{2}{|c|}{ Adjusted OR $(95 \% \mathrm{Cl})$} \\
\hline & Females & Males \\
\hline \multicolumn{3}{|c|}{ Age (years) } \\
\hline 15 & $\mathrm{n} / \mathrm{s}$ & 1 (ref.) \\
\hline 16 & & $4.04(1.29-6.64)^{*}$ \\
\hline 17 & & $3.24(1.14-9.22)^{*}$ \\
\hline 18 & & $3.74(1.39-10.1)^{*}$ \\
\hline \multicolumn{3}{|c|}{ Educational success } \\
\hline Yes & $\mathrm{n} / \mathrm{s}$ & 1 (ref.) \\
\hline No & & $2.89(1.21-6.92)^{*}$ \\
\hline \multicolumn{3}{|c|}{$\begin{array}{l}\text { Having waterpipe user(s) among } \\
\text { family }\end{array}$} \\
\hline No & 1 (ref.) & 1 (ref.) \\
\hline Yes & $3.17(1.70-5.96)^{* *}$ & $3.29(1.57-6.91)^{* *}$ \\
\hline \multicolumn{3}{|c|}{$\begin{array}{l}\text { Having waterpipe user(s) among } \\
\text { friends }\end{array}$} \\
\hline No & 1 (ref.) & 1 (ref.) \\
\hline Yes & $2.19(1.08-4.41)^{*}$ & $4.17(1.82-9.57)^{* *}$ \\
\hline
\end{tabular}

${ }^{*} P \leq 0.05 ;{ }^{* *} P \leq 0.01$.

$\mathrm{n} / \mathrm{s}=$ not significant in model; $\mathrm{OR}=$ odds ratio; $\mathrm{Cl}=$ confidence interval .

strongest predictor of current waterpipe use by male adolescents. A study performed in universities and colleges in the USA showed higher rates of waterpipe smoking among younger students [27]. These observations may be related to social interactions with peers and the process of adolescent socialization. Adolescents are generally looking for opportunities to communicate with their peers, which may make waterpipe use for smoking tobacco attractive and acceptable in this age group.

In the present study, having a waterpipe smoker among family members almost tripled the prevalence of tobacco use in adolescents. This may reflect a lack of close monitoring and the social acceptance of behaviours related to tobacco use, especially the use of waterpipes [25,29]. Similar to previous studies, the results reported here confirmed the impact of tobacco use by a family member on tobacco use by adolescents $[25,32,33]$. A total of $39.7 \%$ of the adolescent respondents predictor of current waterpipe use in boys. Consistent with other studies, this finding shows the association of academic failure with adolescent tobacco use [38]. Although the rate of lifetime waterpipe use was lower ( $51.4 \%$ in boys and $38.9 \%$ in girls) compared with other studies conducted in the Islamic Republic of Iran (64.4\% in boys and 51.3\% in girls), current waterpipe use by adolescents in the country was higher $[21,39]$. Thus, many Iranian adolescents are engaged in waterpipe smoking and are therefore at elevated risk of serious health problems.

Due to several cultural barriers encountered in this study, self-administered questionnaires were used. However, to reduce the under-reporting associated with self-administration and to prevent systematic errors in the study, anonymous questionnaires were used. There were some difficulties in gaining access to the adolescents, especially female respondents, due to parental intervention; however, the parents gave their consent once they had been assured of the confidentiality of the information obtained in the survey. Moreover, this study concentrated only on 4 items related to the pattern of waterpipe use. Further consideration of additional items would yield a better understanding of the patterns of waterpipe use among adolescents. The present study represents the starting point for monitoring the patterns of waterpipe use among Iranian adolescents. Moreover, with regard to oral health, sharing waterpipe use was examined among adolescents and this has not previously been investigated. One of the strengths of this study was the use of demographic information, wealth index, educational status and family relations, all of which are important factors affecting smoking behaviours among adolescents.

In conclusion, the results of the present study performed among Iranian adolescents, which represents the 
first such investigation using a random sample, showed that the experience of waterpipe smoking is common among Iranian adolescents. This study showed that adolescents have a high rate of use of waterpipes for smoking tobacco. Therefore, health promotion programmes should focus on waterpipe use and its social acceptance. Further studies regarding this public health problem are required.
Based on these results, a qualitative study in this field is recommended to explore the views and experiences of adolescent waterpipe smokers. Finally, health promotion programmes focusing on waterpipe use should be developed. As waterpipe use is more socially acceptable than cigarette use in Islamic Republic of Iran $[2,3]$, such prevention programmes must highlight its adverse health effects.
Acknowledgements

We would like to thank all the adolescents and their parents for their participation.

Funding: This project was funded and supported by Tehran University of Medical Sciences; grant no.89-01-2810494.

Competing interests: None declared.

\section{References}

1. Maziak W et al. Adapting smoking cessation interventions for developing countries: a model for the Middle East. International Journal of Tuberculosis and Lung Disease, 2004, 8:403-413.

2. Kandela P. Nargile smoking keeps Arabs in Wonderland. Lancet, 2000, 356:1175.

3. Watad W et al. Water pipe smoking: effects, attitudes and directions. Journal of Smoking Cessation, 2009, 4:18-25.

4. Tamim $\mathrm{H}$ et al. Tobacco use by university students, Lebanon, 2001. Addiction, 2003, 98:933-939.

5. Varsano $\mathrm{S}$ et al. [Waterpipe tobacco smoking among school children in Israel: frequencies, habits, and attitudes.] Harefuah, 2003, 142:736-741 [in Hebrew].

6. Chaaya $\mathrm{M}$ et al. Argileh smoking among university students: a new tobacco epidemic. Nicotine and Tobacco Research, 2004, 6:457.

7. Maziak W et al. Prevalence and characteristics of narghile smoking among university students in Syria. International Journal of Tuberculosis and Lung Disease, 2004, 8:882-889.

8. Refaat A. Practice and awareness of health risk behaviour among Egyptian university students. Eastern Mediterranean Health Journal, 2004, 10:72-81.

9. Zoughaib $\mathrm{S}$ et al. Prevalence and determinants of water pipe or narghile use among students in Beirut's southern suburbs. Le Journal Medical Libanais, 2004, 52:142-148.

10. Momenan AA et al. The rising prevalence of water pipe smoking among Iranian adolescents: Tehran Lipid and Glucose Study. Presentation at the 13th World Conference on Tobacco or Health, Washington DC, 2006.

11. Warren $\mathrm{C}$ et al. Change in tobacco use among 13-15 year olds between 1999 and 2008: findings from the Global Youth Tobacco Survey. Global Health Promotion, 2009, 16(2 Suppl.):38.

12. Primack $B$ et al. Waterpipe tobacco smoking among middle and high school students in Arizona. Pediatrics, 2009, 123:e282.

13. Maziak W. The global epidemic of waterpipe smoking. Addictive Behaviors, 2011, 36:1-5.

14. Kandela P. Signs of trouble for hubble bubble. Lancet, 1997, 349:1460.

15. Shihadeh A, Saleh R. Polycyclic aromatic hydrocarbons, carbon monoxide. Food and Chemical Toxicology, 2005, 43:655-661.

16. Maziak W. The water pipe: time for action. Addiction, 2008, 103:1763-1767.

17. Akl E et al. The effects of waterpipe tobacco smoking on health outcomes: a systematic review. International Journal of Epidemiology, 2010, 39:834-857.
18. How tobacco smoke causes disease: the biology and behavioral basis for smoking-attributable disease: a report of the Surgeon General. Atlanta, Georgia, Centers for Disease Control and Prevention, 2010.

19. Cobb $\mathrm{C}$ et al. Water pipe tobacco smoking: an emerging health crisis in the United States. American Journal of Health Behavior, 2010, 34:275-285.

20. Raad D et al. Effects of water pipe tobacco smoking on lung function: a systematic review and meta-analysis. Chest, 2010, 139(4):764-767.

21. Momenan A et al. Pattern of water pipe (ghalyan) use among intermediate and high school students: a cross-sectional study In Tehran, Iran. Payesh, 2007, 6:135-144.

22. Sabahy A et al. Water pipe tobacco use among Iranian university students: correlates and perceived reasons for use. International Journal of Tuberculosis and Lung Disease, 2011, 15:844-847.

23. 23. Baheiraei $A$ et al. Personal and family factors affecting life time cigarette smoking among adolescents in Tehran (Iran): a community based study. Oman Medical Journal, 2013, 28(3):184-190.

24. Eaton $D$ et al. Youth risk behavior surveillance-United States, 2005. Journal of School Health, 2006, 76:353-372.

25. Warren C. The Global Youth Tobacco Survey Collaborative Group. Tobacco use among youth: a cross country comparison. Tobacco Control, 2002, 11:52-70.

26. Smith $\mathrm{S}$ et al. Harm perception of nicotine products in college freshmen. Nicotine and Tobacco Research, 2007, 9:977.

27. Primack $B$ et al. Prevalence of and associations with waterpipe tobacco smoking among US university students. Annals of Behavioral Medicine, 2008, 36:81-86.

28. Smith-Simone $S$ et al. Water pipe tobacco smoking: knowledge, attitudes, beliefs, and behavior in two US samples. Nicotine and Tobacco Research, 2008, 10:393-398.

29. Mackay J, Amos A. Women and tobacco. Respirology, 2003, 8:123-130.

30. Warren CW. Differences in worldwide tobacco use by gender: findings from the Global Youth Tobacco Survey. Journal of School Health, 2003, 73:207-215.

31. Eaton DK et al. Youth risk behavior surveillance-United States, 2009. Morbidity and Mortality Weekly Report, 2010, 59:1-142.

32. Warren $\mathrm{C}$ et al. Tobacco use by youth: a surveillance report from the Global Youth Tobacco Survey project. Bulletin of the World Health Organization, 2000, 78:868-876.

33. Kelishadi $\mathrm{R}$ et al. Smoking behavior and its influencing factors in a national-representative sample of Iranian adolescents: CASPIAN study. Preventive Medicine, 2006, 42:423-426. 
34. Jackson $\mathrm{C}$ et al. Authoritative parenting, child competencies, and initiation of cigarette smoking. Health Education Quarterly, 1994, 21:103-116.

35. Chassin L et al. Parenting style and smoking-specific parenting practices as predictors of adolescent smoking onset. Journal of Pediatric Psychology, 2005, 30:333-344.

36. Darling N, Steinberg L. Parenting style as context: an integrative model. Psychological Bulletin, 1993, 113:487.

37. Grusec JE, Goodnow JJ. Impact of parental discipline methods on the child's internalization of values: A reconceptualization of current points of view. Developmental Psychology, 1994, 30:4.

38. Bergen HA et al. Perceived academic performance and alcohol, tobacco and marijuana use: longitudinal relationships in young community adolescents. Addictive Behaviors, 2005, 30:1563-1573.

39. Ramezankhani A et al. [Pattern of cigarette smoking in adolescent students in Tehran]. Pejouhandeh, 2010, 15:115-122 [in Farsi]. 